\title{
Reviewer recognition
}

\author{
(c) Springer Nature Limited 2020
}

In this issue we would like to thank all those listed below for taking the time to review for the European Journal of Clinical Nutrition in 2019-your generosity is much appreciated and we hope your association with the journal continues in the future.

\section{Co-Editors-in-Chief: Mario Soares and Manfred Müller}

We want to say a special thanks to the Top 5 Reviewers who gave their time most generously and completed a significant number of reviews for the journal during 2019. We are pleased to offer them a 1-year, gratis online subscription to the European Journal of Clinical Nutrition and Nature.

William Grant, 27
Henry Lukaski, 17
Barbara Boucher, 16

Nevertheless the support of all of you is vital to the journal and we wish to sincerely thank you all too:
Wei He 12

Michael Hermanussen, 11

$\begin{array}{ll}\text { AbuMweis, Suhad S. } & \text { Bhaskaran, Kalpana } \\ \text { Agarwal, Anuja } & \text { Bhatt, Surya } \\ \text { Aguilar, Sheryl } & \text { Binns, Colin } \\ \text { Al Anouti, Fatme } & \text { Biswal, Niranjan } \\ \text { Albrecht, Mathew } & \text { Blewett, Heather } \\ \text { Aldughpassi, Ahmed } & \text { Blundell, John } \\ \text { Altieri, Barbara } & \text { Bogin, Barry } \\ \text { Amrein, Karin } & \text { Bondonno, Catherine } \\ \text { Arora, B } & \text { Bose, Kaushik } \\ \text { Asemi, Zatollah } & \text { Bosy-Westphal, Anja } \\ \text { Attard, Thomas } & \text { Boucher, Barbara } \\ \text { Aubertin-leheudre, Mylene } & \text { Bozzetti, Valentina } \\ \text { Avesani, Carla Maria } & \text { Brand-Miller, Jennie } \\ \text { Balkau, Beverley } & \text { Brandon, L J } \\ \text { Bansal, Manish } & \text { Brett, Neil R. } \\ \text { Baracos, Vickie } & \text { Buchowski, Maciej } \\ \text { Barakatun Nisak, } & \text { Burrows, Raquel } \\ \text { Mohd Yusof } & \text { BW, Teo } \\ \text { Barchetta, Ilaria } & \text { Calder, Philip C } \\ \text { Barclay, Alan } & \text { Calton, Emily } \\ \text { Barker, Margo } & \text { Campbell, Marilyn S. } \\ \text { Begley, Andrea } & \text { Cantone, Elena } \\ \text { Bellisle, France } & \text { Capriati, Teresa } \\ \text { Bellissimo, Nick } & \text { Carberry, Angela } \\ \text { Bergmann, Manuela } & \text { Carey, Sharon } \\ \text { Berry, Sarah } & \text { Carpenter, Catherine } \\ & \end{array}$

Cartmel, Brenda
Carvalho, Kenia
Caulfield, Laura
Cava, Edda
Chan, Dick
Chandak, Giriraj
Chen, Kong
Cheng, Alice
Cheskin, Lawrence
Chiavaroli, Laura
Chidiac, Rony
Clifton, Peter
Colloca, Giuseppe
Connie, Jackaman
Corsinin, Nadia
Crâciun, Elena
Cruz, Adriano G
Cunnane, Stephen
Cunningham, Michael L
Cygan, Heide
Dadhich, J P
Daly, Anne
Dang, Shaonong
Dantas, Roberto
Das Gupta, Rajat
Das, Undurti

Davidson, Jon

De Leeuw, Peter

De Oliveira, Erick

De Zwaan, Martina

Degen, Gisela

Deibert, Peter

Delisle, Helene

Della Pepa, Giuseppe

Di Francesco, Vincenzo

Di Giuseppe, Romina

Dierkes, Jutta

Ding, Rui

Doig, Gordon S.

Donaldson, Michael

Dong, Yanhui

Doola, Raeesa

Doucet, Eric

Drago, Lorenzo

Drake, Isabel

Druml, Wilfred

Dulloo, Abdul

Duriez, Patrick

Ebrahimi-Mameghani,

Mehrangiz

Edwards, Cathrina

El Ghoch, Marwan 
El-Masry, Sahar

Engelke, Klaus

Eshita, Yuki

Fakhoury, Hana

Fayh, Ana Paula

Fernandez, Maria Luz

Ferraro, Manuel

Ferrie, Suzie

Field, Catherine

Forbes, Alastair

Forestell, Catherine

Formoso, Gloria

Fornari, Elena

Forsum, Elisabet

Forsyth, Stewart

Frankenfield, David

Freckmann, Guido

Gaillard, Romy

Gallagher, Emily

Gallaher, Daniel

Gargari, Bahram

Gaskin, Kevin

Gibson, Deanna

Gigante, Denise Petrucci

Gloor, Gregory

Gonzalez, Maria Cristina

Gortan Cappellari, Gianluca

Grant, William

Green, Hilary

Grieger, Jessica

Groen, Bert

Grønbæk, Henning

Gronbaek, Morten

Groopman, John

Gulati, Seema

Gupta, Rajeev

Gupta, Ritesh

Haas, Verena

Hadler, Maria Claret

Haghighian

Roudsari, Arezoo

Haldar, Sumanto

Harinarayan, C V

He, Wei

Hermanussen, Michael

Hettiarachchi, Manjula

Heymsfield, Steven

Hill, Andrew

Hills, Andrew

Hjorth, Mads

Hodson, Leanne

Holick, M F

Hollis, Bruce
Holst, Mette

Holt, Darcy

Holven, Kirsten

Horiuchi, Masahiro

Hosseini-Esfahani, Firoozeh

Huang, Tao

Hulshof, Paul

Hurrell, Richard

Hvas, Christian

Hyer, Warren

Il'yasova, Dora

Iqbal, Khalid

Itkonen, Suvi

Jain, Rishi

James, Anthony

Jan, A

Jensen, Jorgen

Jeppesen, Per

Jésus, Pierre

Jiang, Xinyin

Johnson, Dr Mark

Johnston, Luke

Kabata, Paweł

Kabisch, Stefan

Kampman, Margitta

Karras, Spyridon

Keane, Kevin

Keller, Heather

Kellow, Nicole

Khalatbari-Soltani, Saman

Khan, J

Khan, Tauseef

Khosla, P

Khosla, Pramod

Kim, Rockli

Kluthe, Cheryl

Korpela, Riitta

Koziel, Slawomir

Kroke, Anja

Kruseman, Maaike

Kuriyan, Rebecca

Kurpad, Anura

Kwiecien, Jaroslaw

Lachat, Carl

Lambrichts, Daniel

Lanza, Massimo

Larson, Leila

Laudisio, Daniela

Laurent, Michaël

Laviano, Alessandro

Layden, Brian

Linseisen, Jakob

Liu, Yibin
Llorca, Javier

Lombardo, Mauro

Lottenberg, Ana

Lowe, Michael

Lukaski, Henry

Lukose, Ammu

Ma, Jun

MacLean, William

Madan, Jagmeet

Madill, Janet

Maguire, Donogh

Makarewicz-Wujec,

Magdalena

Martin, Lisa

Masanori, Atsukawa

Massa, Guy

Mattes, Richard D

Maugeri, Andrea

McCarthy, Elaine

McRorie, Johnson

Meade, Anthony

Melton, Phillip

Menon, Purnima

Merli, Manuela

Millward, D Joe

Minisola, Salvatore

Mirmiran, Parvin

Misra, Anoop

Mohan, Viswanathan

Monro, John

Morandi, Anita

Morandi, Antonio

Moretti, Diego

Morgenstern, Jakob

Moukayed, Meis

Mouzaki, Marialena

Mukhopadhyay, Arpita

Musa, Noha

Muthayya, Sumithra

Mutlu, Ece A.

Mwangi, Martin

Nascimento, Gustavo

Neuhäuser-Berthold, Monika

Nezlek, John

Nikniaz, Leila

Nilsen, Dennis

Norman, Richard

Noronha, Jarvis

Nöthlings, Ute

Nowson, Caryl

Nwosu, Benjamin

Ockenga, Johann

Okereke, Ikenna
Orbak, Zerrin

Ozen, Hasan

Ozmen, Hilal Kiziltunc

Pal batthoa, Harjit

Paoli, Antonio

Paturi, Vishnupriya

Pepe, Jessica

Pérez-López, Faustino

Peterson, Sarah

Pfeiffer, Andreas

Pfeiler, Tamara

Philippou, Elena

Pietrobelli, Angelo

Pitocco, Dario

Plank, Lindsay

Pollard, C M

Pontzer, Herman

Pourghassem, Bahram

Powers, Hilary

Prendecka, Monika

Pufulete, Maria

Pugliese, Gabriella

Puri, SeemaRaman, Rajiva

Ramdath, Dan

Rębacz-Maron, Ewa

Remer, Thomas

Rimbach, Gerald

Ritz, Christian

Roberts, Susan

Robertson, M D

Rodriguez, Nancy A.

Romeu Montenegro, Karina

Ross, Catharine

Rossner, Stephan

Roth, Daniel

$\mathrm{Ru}$, Yuan

Rudnev, Sergey

Rush, Elaine

Russo Giellepi, Roasario

Salami, Ali

Salzano, Ciro

Sardinha, Luis

Sarker, Shafiqul

Scalfi, Luca

Schneeweiss, Bruno

Schneider, Stephane

Schoeller, Dale

Schomburg, Lutz

Schrezenmeir, Jürgen

Schumann, Julia

Schwarz, Peter

Schwingshackl, Lukas

Scragg, Robert 
Shao, Jie

Shin, Rumi

Silva, Analiza

Sim, Xueling

Singh, Ankur

Singh, Meenu

Singh, Surya

Sipka, Sandor

Solomons, Noel

Soofi, Sajid

Souza, Nilian Carla

Starkopf, Joel

Stehle, Peter

Stein, Juergen

Stocker, Achim

Stocker, Roland

Stoeving, Renee

Stookey, Jodi

Strand, Tor

Ströbele-Benschop, Nanette

Stubbs, James

Surono, I S

Swaminathan, Sumathi
Szczuko, Małgorzata

Tang, Hong

Tanumihardjo, Sherry

Tasevska, Natasha

Tayyem, Reema

Terranova, Jean

Thankachan, Prashanth

Theis, Stephan

Thomas, Diana

Tieland, Michael

Tome, Daniel

Tong, Liu

Toral, Marta

Torres, Susan

Toteja, Gurdayal

Trautwein, Elke

Tremblay, Angelo

Trembley, Cecilia

Tremellen, Kelton

Tseng, Marilyn

Uusi-Rasi, Kirsti

Vafa, Mohammadreza

Valenzuela, $\mathrm{R}$
Valera-Gran, Desirée

Valerio, Giuliana

Van Goudoever, Johannes B. Winichagoon, Pattannee

Vellanki, Kavitha

Venter, Carina

Verdu, Elena

Verduci, Elvira

Vest, M

Vetrani, Claudia

Vieth, Reinhold

Vikran, Naval

Vitale, Marilena

Von Haehling, Stephan

Wabel, Peter

Walton, Karen

Wang, Guan

Ward, Leigh

Weimann, Arved

Wells, Jonathan

Westenhoefer, Joachim

Westerterp, Klaas

Westerterp-Plantenga,

Margriet
Widhalm, Kurt

Wieringa, Frank

Wirth, Michael

Wirth, Rainer

Wolters, Maike

Woo, Jean

Woodman, Richard

Wu, Yihua

Wuensch, Tilo

Xiao, Su-Mei

Yaseen, A

Ye, Kaixiong

Ye, Weimin

Yokoo, Edna

Zare, Maryam

Zeng, Di

Zhang, Xiaoning

Zhao, C N

Zhao, Xueyin

Zhu, Ruixin

Zhu, Yong 